\title{
Posibles casos de tuberculosis y brucelosis en poblados argáricos de Galera (Granada)*
}

\author{
Possible cases of tuberculosis and brucellosis in Argaric villages in Galera (Granada) \\ Ángel Rubioa, Sylvia A. Jiménez-Brobeil ${ }^{a}$, Lydia P. Sánchez-Barba ${ }^{a}$, Zita Laffranchi ${ }^{a}$ \\ y Fernando Molina ${ }^{b}$
}

\section{RESUMEN}

Una de las características principales de las poblaciones argáricas de la Edad del Bronce de Granada es la existencia de una economía agropecuaria con la presencia de animales dentro de los núcleos poblacionales, lo que supone un riesgo de contagio. En este estudio se presentan unos casos atribuibles a dos enfermedades infecciosas de esta índole hallados en los poblados de Castellón Alto y Fuente Amarga, ambos en Galera (provincia de Granada). En estos yacimientos se han detectado cuatro sujetos que muestran formaciones de hueso nuevo en el tórax (escápulas y costillas) que pueden ser indicativas de tuberculosis y además, en Fuente Amarga, un individuo presenta una lesión característica en la columna vertebral atribuible a brucelosis (epifisitis vertebral). Estos procesos no son de extrañar en poblaciones que tuvieron un contacto estrecho con animales.

\begin{abstract}
One of the main characteristics of Bronze Age Argaric populations of Granada is an agricultural and livestock economy in which animals were present within the settlements. Such presence is a factor in the increase of the risk of contagious diseases. In this study we present some cases that could be linked to animal-transmitted infectious diseases due that have been documented at the sites of Castellón Alto and Fuente Amarga, both located
\end{abstract}

in Galera (province of Granada). At these sites four individuals have been identified with new bone formations in the thorax (scapulae and ribs) that can indicate the presence of tuberculosis. At Fuente Amarga another individual presents a characteristic lesion in the vertebral column linked to brucellosis (vertebral epiphysitis). These features are not uncommon in populations that have close contact with animals.

Palabras clave: Península Ibérica; Edad del Bronce; zoonosis; Tuberculosis; Brucelosis.

Key words: Iberian Peninsula; Bronze Age; zoonosis; tuberculosis; brucellosis.

\section{INTRODUCCIÓN}

El inicio de la agricultura y la ganadería trajo consigo una serie de cambios en el modo de vida de los seres humanos. La pérdida de la movilidad, la estrecha convivencia de humanos y animales dentro del mismo nicho ecológico y el aumento de la población incrementaron el riesgo de propagación de enfermedades infecciosas y parasitarias, entre ellas varias zoonosis, en poblaciones prehistóricas con modelo agropecuario (Cockburn 1971; Barret et al. 1998; Armelagos et al. 2005).

\footnotetext{
* Proyecto "Demografía, dieta y ritual en la Edad del Bronce de los altiplanos granadinos", HAR 2011-27718. Ministerio de Ciencia y Tecnología.

a Laboratorio de Antropología. Facultad de Medicina, Universidad de Granada. Av. de la Investigación 11. 18071 Granada. Correos e.: a_rubiosalvador@hotmail.com orcid.org/0000-0001-6999-5577; jbrobeil@ugr.es orcid.org/0000-0001-8758-5635; lydia.antropologia@hotmail.com orcid.org/0000-0001-7814-0087; zitina82@gmail.com orcid.org/0000-0001-8553-771X.

b Dpto. de Prehistoria y Arqueología. Facultad de Filosofía y Letras. Universidad de Granada. Campus universitario de Cartuja. 18071 Granada. Correo e.: molinag@ugr.es orcid.org/0000-0002-9189-4198.

Recibido 8-IX-2016; aceptado 10-XI-2016.
}

Copyright: (C) 2017 CSIC. Este es un artículo de acceso abierto distribuido bajo los términos de una licencia de uso y distribución Creative Commons Attribution (CC-by) España 3.0. 
Las enfermedades infecciosas constituyen la principal causa de muerte de los seres humanos. Sin embargo, son muy difíciles de diagnosticar sobre el esqueleto dado que su curso rápido impide la formación de lesiones. Las enfermedades infecciosas que causan lesiones específicas sobre el esqueleto son la tuberculosis, la brucelosis, la lepra y las treponematosis (Aufderheide y RodríguezMartín 1998). Las dos primeras se han asociado tradicionalmente con la aparición y desarrollo de la ganadería y sobre ellas se centra este trabajo.

La tuberculosis es una enfermedad infecciosa causada por bacterias del género Mycobacterium que afecta a los mamíferos, humanos y no humanos (Roberts y Buikstra 2003). Dentro del conjunto de organismos que se han denominado como $\mathrm{Myco}$ bacterium tuberculosis complex, son regularmente patógenos para el ser humano el Mycobacterium leprae (causante de la lepra), el Mycobacterium tuberculosis y el Mycobacterium bovis (Aufderheide y Rodríguez-Martín 1998). El M. tuberculosis es transmitido entre humanos por vía respiratoria, debido al contacto de una persona sana con los aerosoles expulsados por una persona infectada. El $M$. bovis se transmite por vía gastrointestinal con el consumo de carne y leche de vacas infectadas. Alternativamente, el contacto de humanos con animales salvajes o domesticados puede dar lugar a la transmisión por ambas vías (Roberts y Buikstra 2003). Las lesiones características sobre hueso se producen en la columna vertebral seguida de las articulaciones de la cadera y la rodilla, aunque también pueden verse afectados los huesos de la mano y el pie, el cráneo (endocráneo), el esternón, las costillas y los huesos largos (Aufderheide y Rodríguez-Martin 1998).

En contexto arqueológico las primeras evidencias de tuberculosis en Europa aparecen en el Neolítico. Figuran casos en Italia (Formicola et al. 1987; Canci et al. 1996), Polonia (Gladykowska-Rzecy 1999; Roberts y Buikstra 2003), Dinamarca (Sager et al. 1972), Hungría (Spekker et al. 2012; Masson et al. 2015; Pósa et al. 2015) y Alemania (Bartels 1907; Steinbock 1976; Nicklisch et al. 2012). De la Edad del Bronce, marco cronológico de este estudio, se han publicado varios casos de tuberculosis en Italia (Canci et al. 2001; Sallustio et al. 2004; Lucatello 2007) y en España (Zamora 1976; Polo et al. 2003).

En la mayoría de los casos publicados, la enfermedad se encuentra muy avanzada y se observa la destrucción y colapso de los cuerpos vertebrales que provocan una cifosis de la columna, lo que se conoce como Mal de Pott (Aufederheide y Rodríguez-Martín 1998). Esta lesión se ha considerado patognomónica de la tuberculosis en Paleopatología. Sin embargo, en numerosas publicaciones se plantea que las lesiones en la cara interna (visceral) de las costillas también podrían ser diagnosticadas como propias de la tuberculosis (Kelley y el Najjar 1980; Kelley y Micozzi 1984; Pfeiffer 1991; Roberts et al. 1994; Matos y Santos 2006; Santos y Roberts 2006; Mariotti et al. 2015).

En la actualidad, el origen de la tuberculosis es muy debatido. La idea tradicional de la evolución de la tuberculosis humana a partir de la enfermedad bovina, muy ligada al proceso de domesticación animal, ha sido muy criticada. Estudios recientes sugieren el origen del $M$. tuberculosis complex hace al menos 2 millones de años en el Este de África (Gutiérrez et al. 2005). Así se establece la hipótesis de la existencia de un $M$. tuberculosis ancestral de tipo humano como cepa original cuya evolución no guardaría relación directa con el M. bovis (Brosch et al. 2002). En este sentido se explicaría la lesión craneal atribuida a tuberculosis en un Homo erectus hallado en Turquía (Kappelman et al. 2008). Incluso se ha planteado que la transmisión pudo ser de humanos a animales domesticados hace al menos 13.000 años con el desarrollo de la agricultura en el Creciente Fértil (Wirth et al. 2008). Sea como fuere, existe una realidad osteológica limitada y los casos más antiguos indiscutibles de tuberculosis en humanos proceden del Neolítico pre-cerámico en el Próximo Oriente (Hershkovitz et al. 2015) y del Neolítico europeo (Formicola et al. 1987; Gladykowska-Rzecycka 1999; Spekker et al. 2012; Masson et al. 2015).

La brucelosis o fiebre de Malta es una enfermedad aguda causada por tres especies de Brucella: Brucella abortus (ganado y caballos), Brucella melitensis (ovejas y cabras) y Brucella suis (cerdo). El microorganismo entra en el cuerpo por la ingestión de la leche y de los productos alimenticios procedentes de animales infectados (Aufderheide y Rodríguez-Martin 1998). A diferencia de la tuberculosis, la infección es zoonótica ya que la transmisión entre humanos es muy rara (Mays 2007). Las lesiones tienen predilección por la columna vertebral donde se observa la erosión 
del ángulo anterosuperior del cuerpo vertebral, denominado Signo de Pedro-Pons (Pedro-Pons 1929).

La brucelosis ha sido menos estudiada que la tuberculosis, lo que se expresa en la escasez de datos en poblaciones prehistóricas europeas. Las primeras evidencias de brucelosis en la Península Ibérica han sido identificadas en yacimientos de época calcolítica, concretamente, en el Dolmen de los Llanos (Etxeberria 1994) y en las Yurdinas II (Gómez 2003), ambos en Álava. A éstos se les suman otras publicaciones en Europa que guardan cierta controversia en el diagnóstico debido a que describían lesiones similares, pero no fueron atribuidas a brucelosis (Curate 2004). Fuera de Europa, en la Edad del Bronce se ha constatado varios casos de brucelosis en el Próximo Oriente (Brothwell 1965; Rashidi et al. 2001; Ortner 2003). Ya en épocas posteriores, para el caso europeo, se ha observado un incremento de esta afección en época romana y medieval debido al aumento de la cría ovina ( $\mathrm{D}^{\prime}$ Anastasio et al. 2011).

La brucelosis pudo afectar a algunos animales salvajes en África, mucho antes de la domesticación, puesto que se ha registrado esta enfermedad en un Australopithecus africanus (2.500.000 de años aprox.) del yacimiento de Sterkfontein en Sudáfrica (D'Anastasio et al. 2009). No obstante el desarrollo de la afección en humanos está estrechamente vinculado con los inicios de la domesticación animal y su aprovechamiento y consumo de productos derivados (Moreno 2014).

Como se ha advertido anteriormente, el registro arqueológico de estas enfermedades en la Península Ibérica es muy limitado. Esto no indica que no tuvieran mayor incidencia en aquellas poblaciones peninsulares con una economía basada en la ganadería y la agricultura. El presente estudio tiene como objetivo la búsqueda de lesiones atribuibles a estas enfermedades para contribuir al conocimiento de su incidencia en la Península Ibérica durante la Edad del Bronce.

\section{YACIMIENTOS}

Los yacimientos de Fuente Amarga y Castellón Alto (Granada) se adscriben a la Edad del Bronce, concretamente, a la conocida como "Cultura de
El Argar" en el Sureste peninsular. Ésta abarca la mayor parte de Andalucía Oriental (Almería, Granada y Jaén), Murcia y el sur de Alicante (Lull 1983). Este fenómeno se caracteriza por poblaciones jerarquizadas con un marcado estatus desde el nacimiento (élites), localización geoestratégica con un urbanismo complejo que incluye sistemas defensivos, sepulturas en el interior del área de habitación con ajuar variado, desarrollo de la metalurgia y una economía agropecuaria (Contreras et al. 1997; Lull 2000; Molina y Cámara 2009; Cámara y Molina 2011).

Los poblados de Castellón Alto y Fuente Amarga pertenecen al término municipal de Galera (Granada) y están a pocos kilómetros entre sí. En esta zona se han documentado una serie de poblados de la Edad del Bronce que se asientan sobre cerros elevados a lo largo del valle de los ríos Castillejar-Galera-Orce (Contreras et al. 1997) (Fig. 1).

La ocupación del poblado y la necrópolis de Castellón Alto tiene lugar desde el Bronce Pleno al inicio del Bronce Tardío (1900-1600 cal ANE) (Molina et al. 2003). El poblado, de pequeñas dimensiones, tiene una posición geoestratégica sobre un espolón que destaca del resto de colinas y un complejo urbanismo establecido en terrazas. En la denominada "acrópolis" situada en la terraza superior vivirían las élites más importantes. En la terraza intermedia se han definido viviendas y espacios de almacenamiento y en la terraza inferior se han registrado estructuras de vivienda (Molina et al. 2003). Por último, en la ladera del cerro contiguo se han localizado otras terrazas con viviendas correspondientes a las fases más recientes de la ocupación. Los enterramientos son en fosas y covachas realizadas en el interior de la vivienda. Ello ha permitido, pese a varios expolios, que la mayoría de los esqueletos presente un excelente estado de conservación.

El yacimiento de Fuente Amarga, muy cercano a Castellón Alto, se ha excavado en menor extensión. Se asienta sobre un cerro y, como el anterior, está dispuesto en terrazas. En las tres terrazas excavadas se han registrado niveles de enterramiento y ocupación. En los trabajos de excavación se hallaron 13 sepulturas de la Edad del Bronce que se encontraban muy alteradas por construcciones de época posterior o por violaciones recientes (Fresneda et al. 1999). Ello conlleva un estado

Trab. Prehist., 74, N. ${ }^{\circ}$ 1, enero-junio 2017, pp. 168-180, ISSN: 0082-5638

doi: $10.3989 /$ tp.2017.12189 

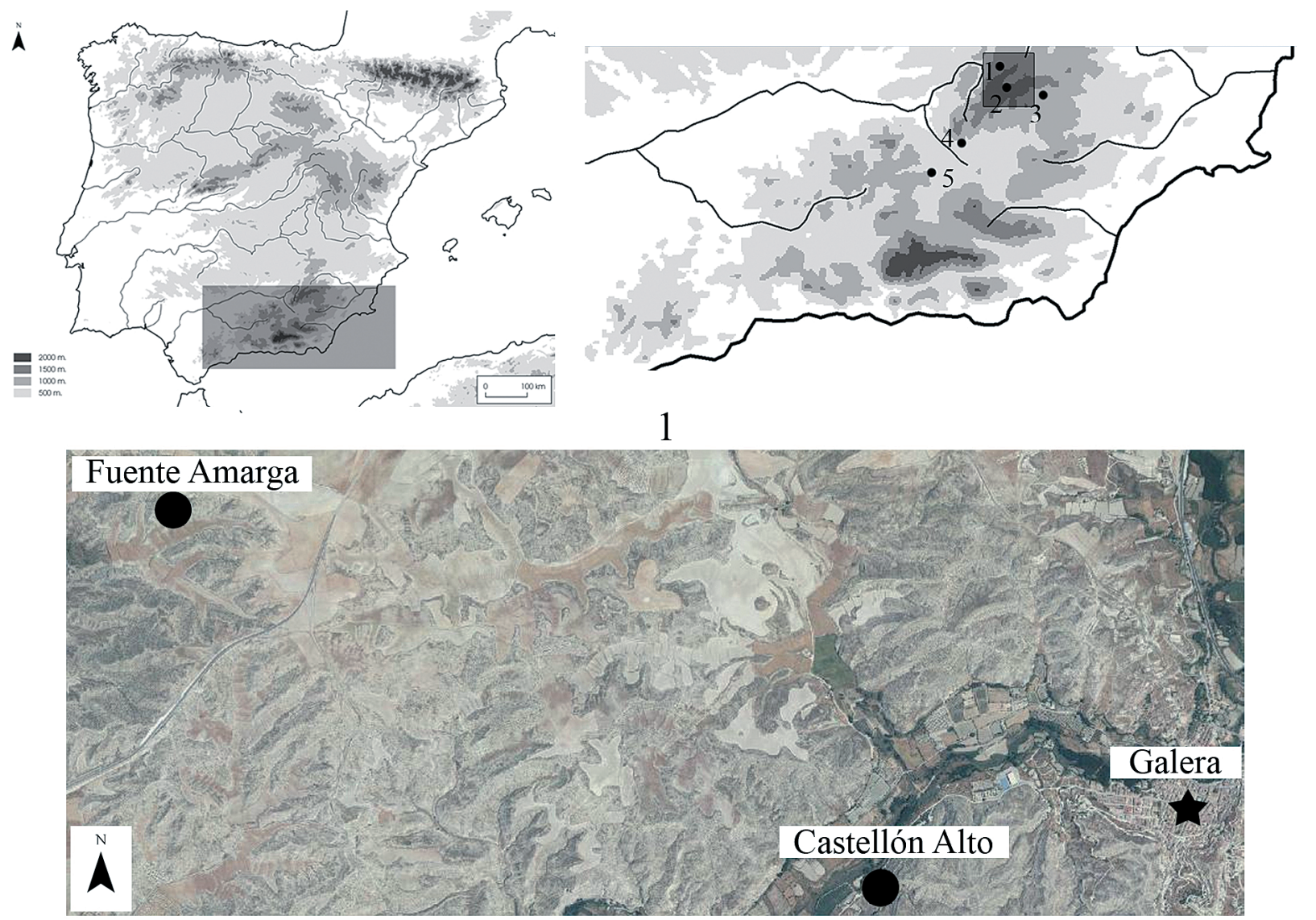

2

Fig. 1. 1. Localización en la Península Ibérica de la región del sureste y situación de los poblados argáricos del estudio: 2. Situación de los yacimientos en el municipio de Galera. Ortofotograma del Plan Nacional de Ortofotografía Aérea (PNOA-2013), Instituto Geográfico Nacional (Madrid).

de conservación muy variable aunque, en líneas generales, el tejido óseo está bien conservado.

\section{MATERIAL Y MÉTODOS}

En la actualidad, los restos humanos que componen ambas poblaciones están depositados en el Laboratorio de Antropología Física perteneciente al Departamento de Medicina Legal, Toxicología y Antropología Física de la Universidad de Granada.

El sexo de los individuos se ha establecido mediante las características morfológicas del cráneo y la pelvis (Ferembach et al. 1979; Byers 2002).
La edad se ha estimado a partir de los cambios observados en la sínfisis púbica para los adultos (Todd 1920) y de los criterios de maduración ósea y erupción dental para los sujetos subadultos (Scheuer y Black 2000; Ubelaker 1984).

Se ha hecho el estudio macroscópico de todas las estructuras óseas de los individuos de ambas poblaciones para detectar aquellas lesiones óseas relacionadas con procesos infecciosos. Para ello se ha descrito minuciosamente la morfología y localización de las mismas, aproximándose a su etiología mediante un diagnóstico diferencial (Aufderheide y Rodríguez-Martín 1998; Ortner 2003; Roberts y Buikstra 2003). Además, la descripción de la epifisitis vertebral ha sido complementada con su estudio radiográfico. 


\section{RESULTADOS}

La colección de Fuente Amarga (FAG) consta de 21 individuos de los cuales cuatro presentan lesiones atribuibles a enfermedades infecciosas. Las lesiones en tres de ellos están en el tórax (Sep. 3a$3 \mathrm{~b}$ y Sep. 15) y son compatibles con tuberculosis.
En el cuarto la alteración indicativa de brucelosis se localiza en el sector lumbar de la columna vertebral (Sep. 9). Entre los 126 individuos de Castellón Alto (CAG) sólo uno (Sep. 58-I) tiene una lesión en el tórax similar a las de Fuente Amarga.

Sepultura 3 (FAG): es un enterramiento múltiple intacto que contiene los restos de 4 indivi-
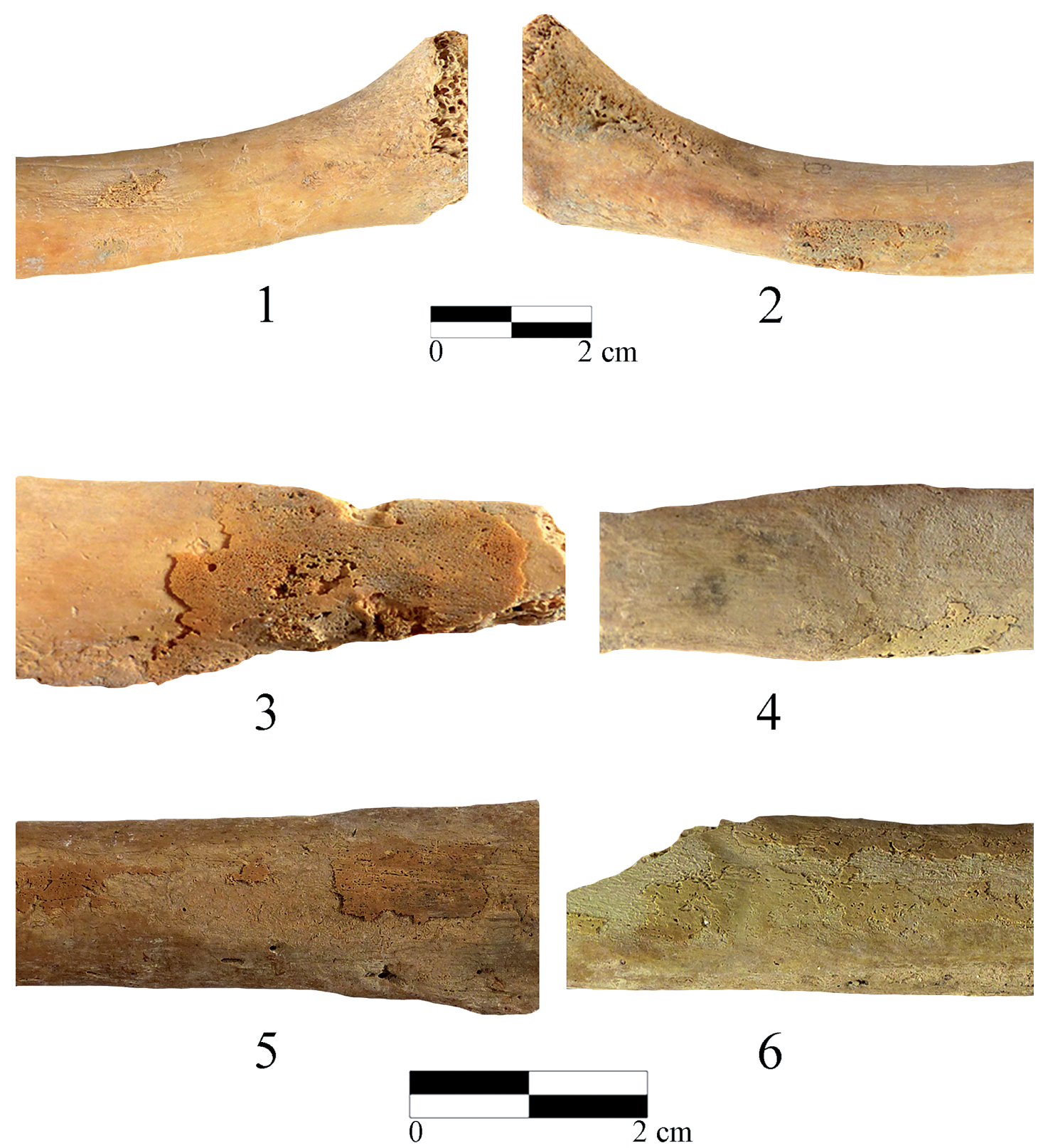

6

Fig. 2. Fuente Amarga (Galera, Granada) Formaciones de hueso nuevo en clavículas y costillas. Sepultura 15: 1.2. vista inferior de clavículas izquierda y derecha, 3. costilla derecha. Sepultura 3: costilla derecha (A), 5.6.: costillas izquierda y derecha (B). 
duos (dos adultos y dos subadultos). Las lesiones han sido identificadas en las costillas de la mujer Madura (A) y del varón Adulto (B). Presentan formaciones de hueso nuevo en la cara visceral de algunas costillas; en ambos casos, la lesión tiene una morfología similar. La mujer tiene afectadas la sexta y séptima costillas derechas en el extremo esternal (Fig. 2: 4). En el varón lo están la sexta costilla izquierda en su extremo esternal y la séptima costilla derecha en su zona central y en su extremo esternal (Fig. 2: 5-6) y, además, conserva otros pequeños fragmentos de costillas del lado izquierdo con formaciones de hueso nuevo.

Sepultura 15 (FAG): está muy alterada y corresponde a un varón juvenil con una edad cercana a los 20 años. Este sujeto tiene formaciones de hueso nuevo en varias localizaciones del esqueleto. Al igual que en la Sepultura 3, presenta aposiciones periósticas en la cara interna de una costilla, en concreto, en el extremo vertebral (cuello) de la quinta derecha (Fig. 2: 3). Además, muestra las mismas lesiones en la cara anterior de la escápula derecha en la zona superior de la fosa subescapular (Fig. 3: 1) y en la región de la inserción del músculo pectoral mayor de ambas clavículas, lo que indicaría una afección bilateral en el tórax (Fig. 2: 1-2).
Sepultura 58-I (CAG): está expoliada. Se pudieron recoger restos de dos sujetos subadultos. Uno es un juvenil con el mismo tipo de lesión en la escápula derecha que el individuo de la Sepultura 15 (FAG). La formación de hueso nuevo en placa aparece en la cara anterior de la escápula y, como ligera diferencia respecto a la lesión del de Fuente Amarga, está situada en la zona cercana al borde lateral de la escápula (Fig. 3: 2).

Sepultura 9 (FAG): es un enterramiento doble intacto que contiene los restos de un varón Maduro y una mujer Adulta. Ésta es quien presenta la lesión. Tiene una edad estimada entre $\operatorname{los} 25$ y 30 años. Sus restos esqueléticos tienen un buen estado de conservación sobre todo en la columna vertebral que tiene todas las vértebras. En la cuarta vértebra lumbar se observa una lesión osteolítica en el borde antero-superior del cuerpo vertebral (Fig. 4: 1). La zona afectada por la lesión presenta una acentuada labiación del borde vertebral caracterizada por una superficie porosa y unos márgenes irregulares con osteofitos (Fig. 4: 2). También la radiografía muestra la labiación en el borde y un aumento de la densidad ósea en el margen inferior de la lesión (Fig. 4: 3). Ni en el resto de la vértebra, ni en las demás vértebras lumbares hay signos patológicos.

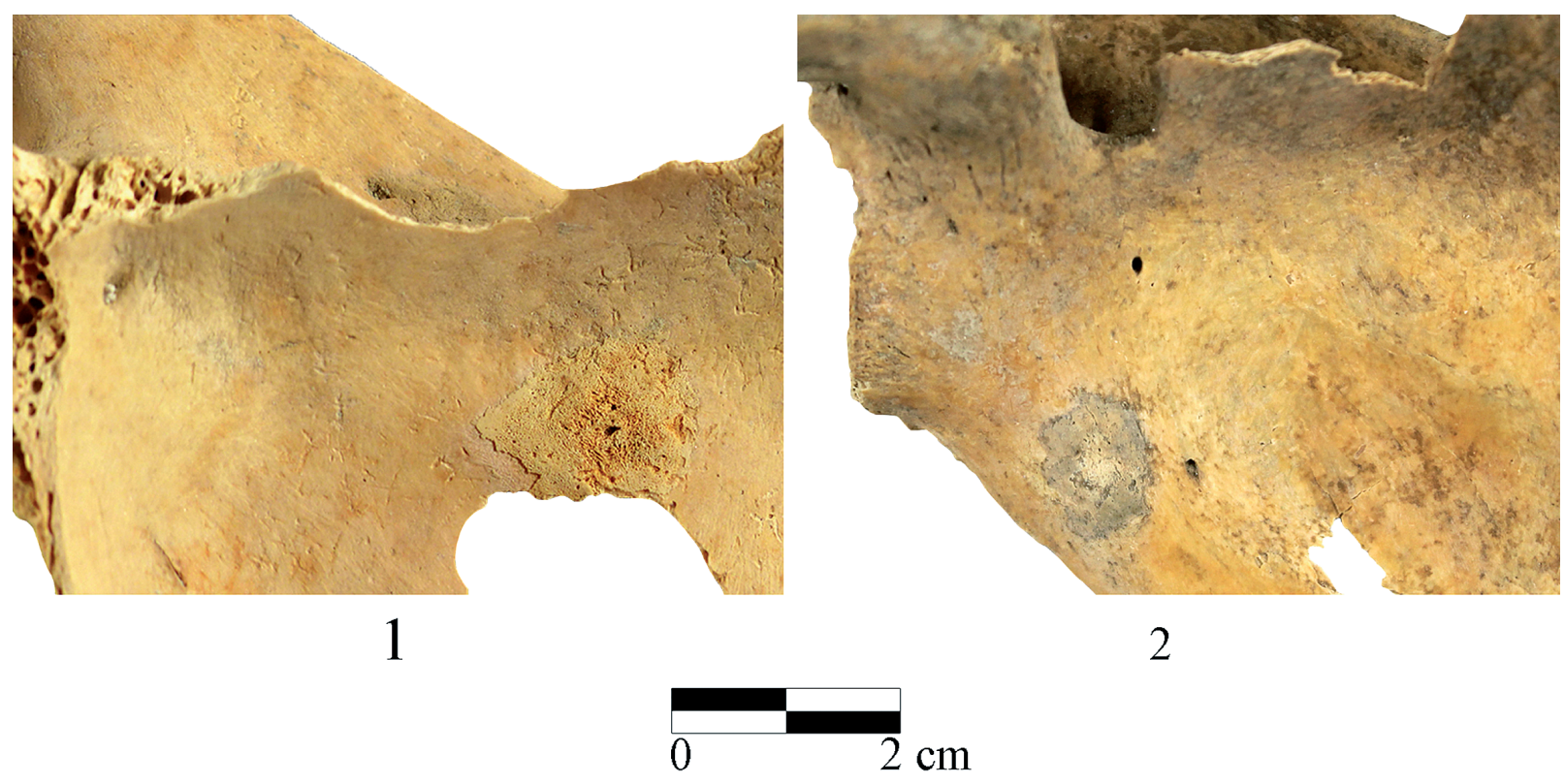

Fig. 3. Formaciones de hueso nuevo en escápulas derechas: 1. Sepultura 15, Fuente Amarga (Galera, Granada); 2. Sepultura 58-I, Castellón Alto (Galera). 

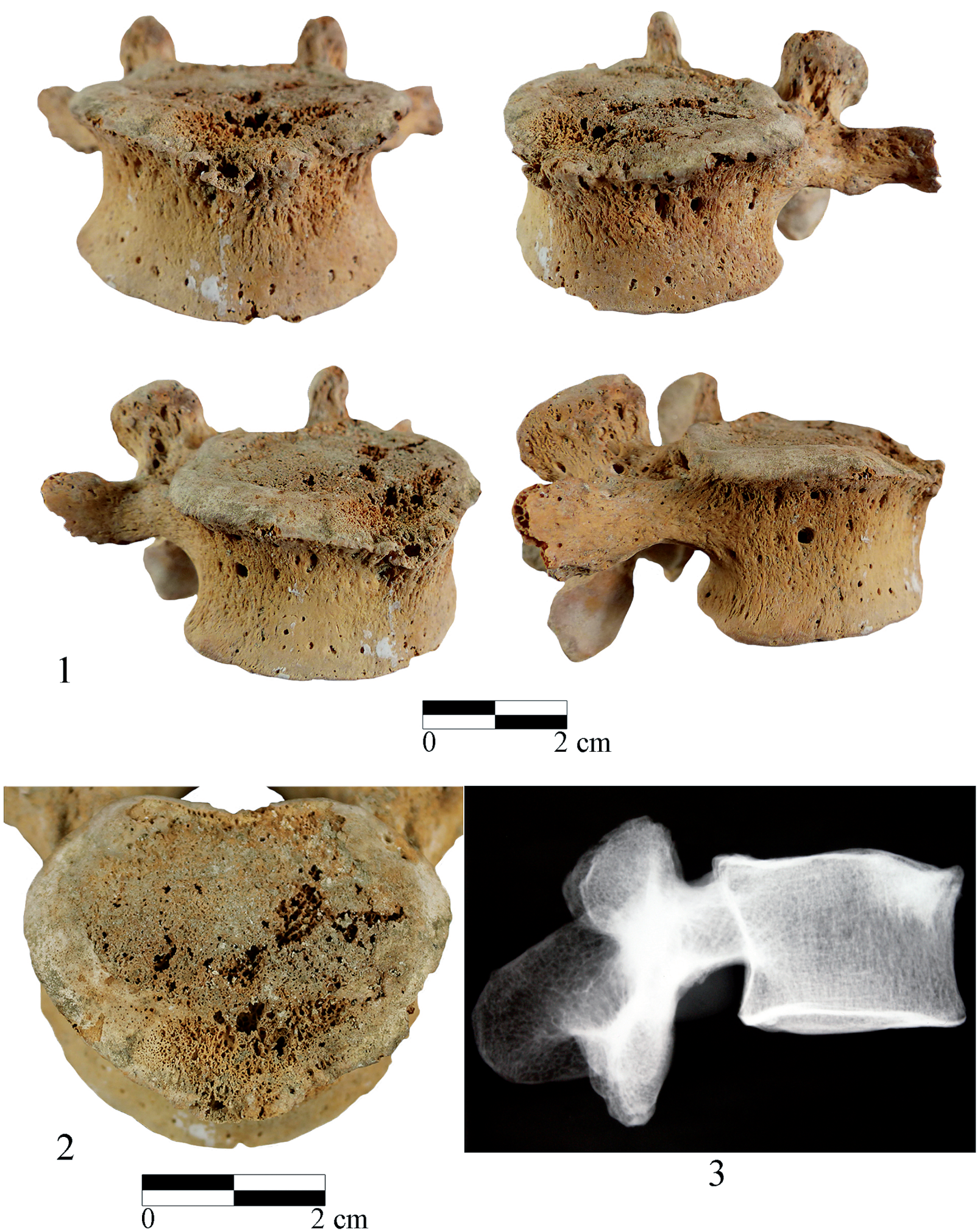

Fig. 4. Epifisitis vertebral (Sepultura 9, Fuente Amarga, Galera, Granada): 1. diferentes vistas de la vértebra, 2. vista de detalle de la zona de la lesión, 3. imagen radiográfica de la vértebra.

Trab. Prehist., 74, N. ${ }^{\circ} 1$, enero-junio 2017, pp. 168-180, ISSN: 0082-5638 doi: $10.3989 /$ tp.2017.12189 


\section{DISCUSIÓN}

La problemática del estudio de las enfermedades infecciosas en Paleopatología radica en dos puntos fundamentales. La afirmación del diagnóstico queda restringida a los casos donde la lesión está muy desarrollada y se considera suficientemente identificativa, o a los que permiten confirmar la enfermedad mediante otro tipo de análisis. En segundo lugar, al tratarse de poblaciones de procedencia arqueológica, la conservación es variable y no siempre óptima, más aún si se trata de costillas o vértebras que son muy frágiles $y$, por ello, más susceptibles a los procesos tafonómicos.

\subsection{Tuberculosis}

La morfología de las lesiones localizadas en el tórax (escápula, clavícula o costillas) es homogénea en los individuos argáricos. Todas son formaciones de hueso nuevo en placa donde se observa claramente la separación entre la cortical del hueso y la aposición de hueso nuevo. En las últimas décadas, se han documentado formaciones de hueso nuevo en la superficie visceral de las costillas en colecciones de sujetos que padecieron tuberculosis (Kelley y el Najjar 1980, El-Najjar 1981; Kelley y Micozzi 1984; Pfeiffer 1991; Roberts et al. 1994; Santos y Roberts 2006; Matos y Santos 2006; Mariotti et al. 2015). Hay que ser prudente a la hora de asociar estas lesiones costales con tuberculosis para poblaciones antiguas ya que de por sí no son determinantes (Mays et al. 2002). Sin embargo, pese a no ser signos patognomónicos de tuberculosis, se consideran indicadores no específicos de infección crónica pulmonar. La inflamación pleural podría producir una reacción perióstica dando lugar a formaciones de hueso nuevo en la cara interna de las costillas y por ello, no son descartables otras afecciones pulmonares como la neumonía, bronquitis, enfisema o pleuresía (Roberts et al. 1994).

Ahora bien, la morfología de la lesión y su localización en la caja torácica puede proporcionar más datos para el diagnóstico de la tuberculosis pulmonar (Santos y Suby 2012). Se ha observado en individuos con tuberculosis que las formaciones de hueso nuevo afectan con mayor frecuencia a las costillas superiores y medias de la caja torácica (Santos y Roberts 2006; Matos y Santos 2006), como se vio en los individuos argáricos analizados. La ocurrencia suele ser bilateral (Matos y Santos 2006), como le ocurre al varón de la Sepultura 3 (FAG) y al juvenil de la Sepultura 15 (FAG).

Los casos de la Sepultura 15 (FAG) y la Sepultura 58-I (CAG) presentan formaciones de hueso nuevo en la cara anterior de la escápula, localizaciones, al igual que en las clavículas (S15, FAG) ya observadas en individuos con tuberculosis (Matos y Santos 2006). Además, guardan gran similitud con las lesiones, atribuidas a esta infección, en sujetos pertenecientes a la "Cultura del Bronce Valenciano" de la Cova dels Blaus (Polo et al. 2003). Bien es cierto que, en casos avanzados de osteoartropatía hipertrófica (OHP), además de la afección en los huesos tubulares (fíbulas, tibias, ulnas y radios), pueden aparecer formaciones de hueso nuevo en clavículas y escápulas (Ortner 2003). Estas lesiones en las extremidades, compatibles con la OHP, fueron observadas en individuos que padecieron tuberculosis (Assis et al. 2011). Sin embargo, los argáricos estudiados no presentan afectadas sus extremidades.

Estas lesiones en sí mismas no son determinantes. Sin embargo análisis biomoleculares recientes, aplicados en poblaciones de origen arqueológico para confirmar la presencia de tuberculosis, tuvieron resultados afirmativos. Cabe destacar que individuos neolíticos en Hungría (Masson et al. 2015) y Alemania (Nicklisch et al. 2012) mostraban formaciones de hueso nuevo en la cara visceral de las costillas, similares a las del presente estudio.

\subsection{Brucelosis}

La lesión observada en la vértebra lumbar (L4) de la mujer adulta de la Sepultura 9 corresponde a una epifisitis vertebral. Su origen puede ser la brucelosis pero también otras enfermedades como la tuberculosis, la enfermedad de Scheuermann, la osteomielitis piógena y las lesiones discales, entre otras (Aufderheide y Rodríguez-Martín 1998; Ortner 2003).

Debido a la presencia de individuos en Fuente Amarga que pudieron padecer tuberculosis y a 
que ésta afecta también a la columna vertebral, se hace necesaria la distinción. La tuberculosis, a diferencia de la brucelosis, suele causar abscesos en las vértebras, colapso vertebral y cifosis de la columna (Aufderheide y Rodríguez-Martín 1998), y la mujer de Fuente Amarga no muestra estos signos. La enfermedad de Scheuermann produce epifisitis vertebral pero usualmente ocurre en el sector dorsal involucrando a más vértebras y la lesión es muy distinta, ya que afecta a varias zonas de la misma vértebra. También la osteomielitis piógena, aunque tiene predilección en el sector lumbar, causa lesiones líticas en los cuerpos vertebrales, aunque suele involucrar otras partes de la vértebra. En cambio, la mujer de la Sepultura 9 presenta una lesión muy focalizada en el borde superior del cuerpo vertebral y carece de señales patológicas en el resto de la columna. Por último, la herniación anterior del disco intervertebral produciría una lesión focalizada en la misma zona y con una morfología relativamente parecida como ha sido observado en otro estudio (Mays 2007). La mujer no supera los 30 años de edad y es poco probable que este tipo de lesiones discales aparezcan en una edad tan temprana y sin ir acompañadas de otros indicadores degenerativos en la columna vertebral. Este caso en particular muestra elementos identificativos que apuntarían a la brucelosis como causa más probable. Por un lado, la lesión se restringe a la zona del annulus fibrosus con unos márgenes bien definidos. En su borde anterior se advierte la formación de osteofitos, algo usual debido al proceso de restauración propio de los casos de brucelosis (Etxeberria 1994). Por otro lado, el aumento de la porosidad en la zona central de la lesión disminuye hacia los márgenes y, asimismo, en la cara anterior del cuerpo vertebral se ven ligeros inicios de formación de hueso nuevo, una característica ya señalada en la población de Herculano (Capasso 1999). Por último, la radiografía muestra un aumento de la densidad ósea justo en la zona inferior de la lesión osteolítica, debido a la reacción esclerótica característica de la brucelosis (Pedro-Pons 1929).

La escasez de datos tanto a nivel general como dentro de la Península Ibérica limita la comparación de la lesión con otros casos confirmados como brucelosis. Únicamente se ha señalado la presencia de brucelosis en un individuo del Calcolítico peninsular (Etxeberria 1994). La afectación osteológica no refleja el papel que, en realidad, pudo jugar esta enfermedad en las primeras poblaciones ganaderas. Si la historia de esta enfermedad está muy potenciada por el desarrollo de la domesticación animal, pudo afectar a poblaciones más recientes, como las argáricas de la Edad del Bronce, máxime cuando su economía se sustentaba en gran parte en la ganadería.

\subsection{Actividades ganaderas}

El riesgo de contraer las enfermedades infecciosas citadas depende en gran parte de las condiciones de vida que pueden disminuir o aumentar el riesgo de contagio. Las poblaciones asentadas en el Castellón Alto y Fuente Amarga tienen una economía basada en la agricultura y la ganadería, que establecería los animales domesticados como una de las fuentes de infección humana. En Castellón Alto se han documentado zonas o áreas de estabulación (Moreno y Haro 2008) y en Fuente Amarga el análisis faunístico determinó la presencia sobre todo de ovicápridos $\mathrm{y}$, en menor medida, de ganado bovino (Fresneda et al. 1999). Como hecho representativo, en gran parte de las necrópolis argáricas, incluyendo las de este estudio, aparecen ajuares cárnicos en el interior de las sepulturas que han sido interpretados como rituales de comensalidad (Aranda y Esquivel 2006; Aranda y Esquivel 2007).

El aprovechamiento alimenticio del ganado por estas poblaciones, ya sea cotidiano o como parte del ritual funerario, podría establecer un origen zoonótico de la tuberculosis por ingesta de carne o leche de vaca infectada. Sin embargo, no se puede descartar que la infección fuese transmitida entre humanos. En cambio, la brucelosis al ser de contagio zoonótico (animal-humano) sólo pudo transmitirse por el consumo de leche o productos procedentes, seguramente, de ovicápridos.

\subsection{Aspectos poblacionales}

Las dificultades intrínsecas que subyacen en este tipo de estudios limita la posibilidad de arrojar nuevos datos sobre las condiciones de vida de las poblaciones argáricas. La primera es la visión sesgada de la realidad demográfica derivada de 
la excavación parcial de los yacimientos. En segundo lugar, la detección de estas enfermedades depende de la representación osteológica conservada de la colección de individuos exhumados. La tercera dificultad se haya en el restringido número de sujetos que muestran estos signos patológicos concretos. Teniendo en cuenta lo anterior, los sujetos están afectados por la tuberculosis con independencia del sexo y la edad. Además no se puede establecer la edad de contagio de los individuos ya que depende del propio progreso de la infección, es decir, de la carga de patógenos y su virulencia y de la respuesta inmune del hospedador. Hay que destacar que sólo un porcentaje reducido de los enfermos de tuberculosis activa desarrolla lesiones en el esqueleto (Steinbock 1976). Ello significa que pudo afectar a más sujetos de las dos poblaciones arqueológicas estudiadas que, al no presentar señales en sus huesos, pasaron inadvertidos.

Cabe recordar que las poblaciones argáricas tienen una esperanza de vida baja (Jiménez et al. 2000) y que los posibles afectados podrían haber muerto antes de desarrollar lesiones óseas. La muerte temprana de los sujetos explicaría por qué no han aparecido las lesiones propias de estadios más avanzados de la enfermedad (Baker et al. 1999). En todo caso, los cuatro individuos sí desarrollaron formaciones de hueso nuevo, indicativas de que padecieron la enfermedad durante un periodo prolongado. Ello descartaría otras afecciones pulmonares que provocan la muerte del sujeto en un espacio relativamente corto de tiempo.

No se puede asegurar de cuál de las dos poblaciones procede la infección, ni descartar que proviniera de otra distinta. Para que la enfermedad apareciera en individuos de ambas poblaciones o éstas eran coetáneas o aquella tuvo un origen paralelo. Ahora bien, la cercanía entre ambos yacimientos permite suponer que el contacto entre ambos grupos (personas y animales) explicaría la propagación de un núcleo poblacional a otro. Es cierto que en Castellón Alto, donde se han excavado numerosas sepulturas y que parece ser un núcleo amplio de población, habría más posibilidades de contagio que en Fuente Amarga, donde se han documentado tres individuos. La diferencia de afección puede no radicar en el número de sujetos que componen la población sino en su composición familiar: en un ámbito más cerrado habría mayor contacto entre los individuos y, por ello, unas circunstancias ideales para la propagación de este tipo de enfermedades.

No se debe olvidar que las enfermedades infecciosas, como la tuberculosis o la brucelosis, afectan a los individuos al margen de su estatus social, como manifiesta el ajuar de las sepulturas. La Sepultura 3 de Fuente Amarga presenta el ajuar más rico de las excavadas en el yacimiento frente al del resto de las tumbas, entre ellas el de la Sepultura 15, más modesto. Sin embargo, ambas sepulturas albergan sujetos que pudieron padecer tuberculosis. En definitiva, las diferencias sociales, atestiguadas en la cultura argárica, no implican unas condiciones de vida diametralmente opuestas que marquen diferencias a la hora de la susceptibilidad a padecer enfermedades infecciosas. La singularidad del caso de brucelosis de Fuente Amarga, más allá de la propia relevancia de su presencia en la Edad del Bronce, no aporta mayor información en cuanto a las implicaciones sociales de la enfermedad.

\section{CONCLUSIONES}

El estudio de las poblaciones argáricas de Granada (Castellón Alto y Fuente Amarga) ha evidenciado la existencia de individuos que pudieron padecer enfermedades infecciosas específicas como la tuberculosis y la brucelosis. Las lesiones identificadas en el tórax pueden no bastar por sí mismas para afirmar la presencia de tuberculosis. Ahora bien, las formaciones de hueso nuevo en la cara visceral de las costillas y en otras localizaciones del tórax son marcadores no específicos de enfermedad pulmonar. Salvando las limitaciones de este tipo de estudios en poblaciones de origen arqueológico, hay que valorar que no se trata de un caso singular sino de varios individuos que presentan estas lesiones, lo que atestigua una enfermedad infecciosa. Además, su localización y morfología ya ha sido constatada en otras series de individuos con tuberculosis. En definitiva, la tuberculosis pudo ser la enfermedad que afectó a estos individuos granadinos. Para este caso no se puede establecer un origen en los animales domesticados ya que la tuberculosis puede transmitirse entre humanos aunque podría ser la causa probable. Por el contrario, la mujer de Fuente Amarga 
cuya columna vertebral tiene signos indicativos de brucelosis es muestra evidente de la infección, relacionada con los animales domesticados y su aprovechamiento. La realidad argárica demuestra una estrecha convivencia entre humanos y animales que intensificaría el riesgo de contagio.

Finalmente, no hay que infravalorar el papel que juegan este tipo de enfermedades en poblaciones prehistóricas cuya economía radica en la agricultura y la ganadería. No es de extrañar que estén presentes tanto en el mundo argárico como en contextos más tempranos. A ese respecto el número reducido de casos registrados en la Península Ibérica no excluye que la incidencia de este tipo de enfermedades pudiera haber sido mayor.

\section{BIBLIOGRAFÍA}

Aranda, G. y Esquivel, J. A. 2006: "Ritual funerario y comensalidad en las sociedades de la Edad del Bronce del sureste peninsular: La Cultura de El Argar". Trabajos de Prehistoria 63(2): 117-133.

Aranda, G. y Esquivel, J. A. 2007: "Poder y prestigio en las sociedades de la Cultura de El Argar. El consumo comunal de bóvidos y ovicápridos en los rituales de enterramiento". Trabajos de Prehistoria 64(2): 95-118.

Armelagos, G. J.; Brown, P. J. y Turner, B. 2005: "Evolutionary, historical and political economic perspectives on health and disease". Social Science \& Medicine 61(4): 755-765.

Assis, S.; Santos, A. L. y Roberts, C. 2011: "Evidence of hypertrophic osteoarthropathy in individuals from the Coimbra Skeletal Identified Collection (Portugal)". International Journal of Paleopathology 1: 155-163.

Aufderheide, A. y Rodríguez-Martín, C. 1998: The Cambridge enciclopedia of human paleopathology. Cambridge University Press. Cambridge.

Baker, B. J. 1999: "Early manifestations of tuberculosis in the skeleton". En G. Y. Pálfi, O. Dutour,J. Deak y I. Hutás (eds.): Tuberculosis: past and present. Golden Book Publishers and Tuberculosis Foundation. Budapest-Szeged: 301-310.

Barrett, R.; Kuzawa, C. W.; McDade, T. y Armelagos, G. J. 1998: "Emerging and reemerging infectious diseases: the third epidemiologic transition". Annual review of anthropology 27: 247-271.

Bartels, P. 1907: "Tuberkulosein der Jtingeren Steinzeit". Archiv ftir Anthropologie 6: 243-255.

Brosch, R.; Gordon, S. V.; Marmiesse, M.; Brodin, P.; Buchrieser, C.; Eiglemeier, K., Garnier, T.; Gutiérrez, C.; Hewinson, G., Kremer, K., Parsons, L.
M.; Pym, A. S.; Samper, S.; Van Soolingen, D. y Cole, S. T. 2002: "A new evolutionary scenario for the Mycobacterium tuberculosis complex". Proccedings of the National Academy of Sciences 99(6): 3684-3689.

Brothwell, D. 1965: "The paleopathology of early Middle Bronze Age remains from Jericho". Jericho 2: 585-693.

Byers, S. N. 2002: Introduction to forensic Anthropology. Allyn and Bacon. Boston.

Cámara, J. A. y Molina, F. 2011: “Jerarquización en el mundo argárico (2000-1300 aC)". Quaderns de Prehistoria i Arqueologia de Castellò 29: 77-104.

Canci, A.; Minozzi, S. y Borgognini Tarli, S. M. 1996: "New evidence of tuberculous spondylitis from Neolithic Liguria (Italy)". International Journal of Osteoarchaeology 6: 497-501.

Canci, A.; Minozzi, S. y Borgognini Tarli, S. M. 2001: "Tuberculosis spondylitis during the Bronze Age: two cases from Italy". En M. La Verghetta y L. Capasso (eds.): Proceedings $13^{\text {th }}$ European Meeting of the Paleopathology Association (Chieti, Italia 2001): 67-71. Teramo, Italia.

Capasso, L. 1999: "Brucellosis at Herculaneum (79 AD)". International Journal of Osteoarchaeology 9: $227-288$.

Cockburn, T. A. 1971: "Infectious diseases in ancient populations". Current Anthropology 12(1): 45-62.

Contreras, F.; Rodríguez-Ariza, M. O.; Cámara, J. A. y Moreno, A. 1997: Hace 4000 años: Vida y muerte en dos poblados de la Alta Andalucía. Catálogo de la exposición. Junta de Andalucía-Fundación Caja de Granada. Granada.

Curate, F. 2004. "A brucelose em paleopatologia: um estudo de caso proveniente da necrópole Cristã de Cacela Velha". Antropologia Portuguesa 20-21: 209-235.

D’Anastasio, R.; Stanisca, T.; Milia, M. L.; Manzoli, L. y Capasso, L. 2011: "Origin, evolution and paleoepidemiology of brucellosis". Epidemiology and infection 139(1): 149-156.

D’Anastasio, R.; Zipfel, B.; Moggi-Cecchi, J.; Stanyon, R. y Capasso, L. 2009: "Possible brucellosis in an early hominin skeleton from Sterkfontein, South Africa". PLoS One 4(7): 1-5.

El-Najjar, M. 1981: "Skeletal changes in tuberculosis: the Hamann-Todd Collection". En J. Buikstra (ed.): Prehistoric tuberculosis in the Americas. Northwestern University Archaeological Program. Chicago: 85-97.

Etxeberria, F. 1994: "Vertebral epiphysis: early signs of brucellar disease". Journal of Paleopathology 6(1): 41-49.

Ferembach, D.; Schwidetzky, I. y Stloukal, M. 1979: "Recommandations pour determiner l'âge et le sexe sur le squelette". Bulletin et Mémoires de la Société d'Anthropologie de Paris 6(XIII): 7-45.

Fresneda, E.; Rodríguez M. O.; López, M. y Peña, J. M. 1999: "El asentamiento argárico de Fuente Amarga

Trab. Prehist., 74, N. ${ }^{\circ} 1$, enero-junio 2017, pp. 168-180, ISSN: 0082-5638

doi: $10.3989 /$ tp.2017.12189 
(Galera, Granada)". En Actas del XXIV Congreso Nacional de Arqueología (Cartagena 1997): 231240. Murcia.

Formicola, V.; Milanesi, Q. y Scarsini, C. 1987: "Evidence of spinal tuberculosis of the 4th millennium BC from Arene Candide Cave (Liguria, Italy)". American Journal of Physical Anthropology 72: 1-6.

Gladykowska-Rzeczycka, J. 1999: "Tuberculosis in the past and present in Poland". En G. Pálfi, O. Dutour, J. Deak y I. Hutás (eds.): Tuberculosis: past and present. Golden Book Publishers and Tuberculosis Foundation. Budapest-Szeged: 561-576.

Gómez, A. 2003: "Los restos humanos. Análisis de los restos morfológicos". En J. Fernández (ed.): Las Yurdinas II: un depósito funerario entre finales del IV y comienzos del III milenio BC. Memorias de yacimientos alaveses 8, Diputación Foral de Álava. Vitoria-Gasteiz: 109-126.

Gutiérrez, M. C.; Brisse, S.; Brosch, R.; Fabre, M.; Omais, B.; Marmiesse, M.; Supply, P. y Vincent, V. 2005: "Ancient origin and gene mosaicism of the progenitor of Mycobacterium tuberculosis". PLos Pathogens 1: e5.

Hershkovitz, I.; Donoghue, H. D.; Minnikin, D. E.; May, H.; Lee, O. Y. C.; Feldman, M.; Galili, E.; Spigelman, M.; Rothschild, B. M. y Bar-Gal, G. K. 2015: "Tuberculosis origin: The Neolithic scenario". Tuberculosis 95(Supplement 1): 122-126.

Jiménez-Brobeil, S.; Botella, M. y Alemán, I. 2000: "Las poblaciones argáricas. Apuntes paleodemográficos”. En L. Caro, H. Rodríguez, E. Sánchez, B. López y M. J. Blanco: Tendencias actuales de investigación en la Antropología Física española. Secretariado de publicaciones, Universidad de León. León: 199-204.

Kappelman, J.; Alçiçek, M. C.; Kazanci, N.; Schultz, M.; Özkul, M. y Şen, S.. 2008: "First Homo erectus from Turkey and implications for migrations into températe Eurasia". American Journal of Physical Anthropology 135(1): 110-116.

Kelley, M. A. y El Najjar, M. Y. 1980: "Natural variation and differential diagnosis of skeletal changes in tuberculosis". American Journal of Physical Anthropology 52: 153-167.

Kelley, M. A. y Micozzi, M. S. 1984: "Rib lesions in chronic pulmonary tuberculosis". American Journal of Physical Anthropology 65: 381-386.

Lucatello, F. 2007: La tubercolosi ossea: casi di studio dalla necropoli dell'eta del Bronzo media e recente di Olmo di Nogara (VR). Padova University. Padua.

Lull, V. 1983: La Cultura de El Argar, un modelo para el estudio de las formaciones económico-sociales prehistóricas. Akal. Madrid.

Lull, V. 2000: "Argaric society: death at home". Antiquity 74(285): 581-590.

Mariotti, V.; Zuppello, M.; Pedrosi, M. E.; Bettuzzi, M.; Brancaccio, R.; Peccenini, E.; Morigi, M. P. y Belcastro, M. G. 2015: "Skeletal evidence of tu- berculosis in a modern identified human skeletal collection (Certosa Cementery, Bologna, Italy)". American Journal of Physical Anthropology 157: 389-401.

Masson, M.; Bereczki, Z.; Molnár, E.; Donoghue, H. D.; Minnikin, D. E.; Lee, O. Y. C.; Wu, H. H. T.; Besra, G. S.; Bull, I. D. y Pálfi, G. 2015: “7000 year-old tuberculosis cases from Hungary-Osteological and biomolecular evidence". Tuberculosis 95(Supplement 1): 13-17.

Matos, V. y Santos, A. L. 2006: "On the trail of pulmonary tuberculosis base on rib lesions: Results from the human identified skeletal Collection from the Museu Bocage (Lisbon, Portugal)". American Journal of Physical Anthropology 130(2): 190-200.

Mays, S. 2007: "Lysis at the anterior vertebral body margin: Evidence for brucellar spondylitis?". International Journal of Osteoarchaeology 17: 107-118.

Mays, S.; Fysch, E. y Taylor, G. M. 2002: "Investigation of the link between visceral surface rib lesions and tuberculosis in a medieval skeletal series from England using ancient DNA". American Journal of Physical Anthropology 119: 27-36.

Molina, F. y Cámara, J. A. 2009: "La cultura argárica en Granada y Jaén”. En M. Hernández, J. A. Soler y J. A. López (eds.): En los confines del Argar. Una cultura de la Edad del Bronce en Alicante. Museo Arqueológico de Alicante. Alicante: 196-223.

Molina, F.; Rodríguez-Ariza, M. O.; Jiménez, S. y Botella, M. 2003: "La sepultura 121 del yacimiento argárico de el Castellón Alto (Galera, Granada)". Trabajos de Prehistoria 60(1): 153-158.

Moreno, E. 2014: "Retrospective and prospective perspectives on zoonotic brucellosis". Frontiers in $\mathrm{Mi}$ crobiology 5: 213.

Moreno, A. y Haro, M. 2008: “Castellón Alto (Galera, Granada). Puesta en valor de un yacimientoargárico". Cuadernos de Prehistoria y Arqueología de la Universidad de Granada 18: 371-395.

Nicklisch, N.; Maixner, F.; Ganslmejer, R.; Friederich, S.; Dresely, V.; Meller, H.; Zink, A. y Alt, K. W. 2012: "Rib lesions in skeletons from early Neolithic sites in central Germany: On the trail of tuberculosis at the onset of agriculture". American Journal of Physical Anthropology 149: 391-404.

Ortner, D. 2003: Identification of pathological conditions in human skeletal remains. Academic Press. New York.

Pedro-Pons, A. 1929: "La espondilitis melitococica". Annals de Medicina 23: 227-236.

Pfeiffer, S. 1991: "Rib lesions and New World tuberculosis". International Journal of Osteoarchaeology 1: 191-198.

Polo, M.; Casabó, J.; Puchalt, F. y Villalaín, J. D. 2003: "Probables evidencias de tuberculosis en el bronce valenciano: Cova dels Blaus (Vall d'Uixó, Caste1ló)". En A. Cañellas (ed.): Nuevas perspectivas del diagnóstico diferencial en Paleopatología. Actas del 
VII Congreso Nacional de Paleopatología (Mahón, Menorca 2003): 244-257. Mahón.

Pósa, A.; Maixner, F., Gusztáv Mende, B.; Köhler, K., Osztás, A.; Sola, C.; Dutour, O.; Masson, M.; Molnár, E.; Pálfi, G. y Zink, A. 2015: "Tuberculosis in Late Neolithic-Early Copper Age human skeletal remains from Hungary. Tuberculosis 95(Suplement 1): $18-22$.

Rashidi, J. S.; Ortner, D. J.; Frohlich, B. y Jonsdottir, B. 2001: "Brucellosis in early Bronze age Jordan and Bahrain: an analysis of posible cases of Brucella spondylitis". American Journal of Physical Anthropology 114: 122.

Roberts, C. A. y Buikstra, J. E. 2003: The bioarchaeology of tuberculosis: a global view on a reemerging disease. University Press of Florida. Gainesville.

Roberts, C. A.; Lucy, D. y Manchester, K. 1994: "Inflammatory lesions of ribs: an analysis of the Terry Collection". American Journal of Physical Anthropology 95: 169-182.

Sager, P.; Schalimtzek, M. y Moller-Christensen, V. 1972: "A case of spondylitis tuberculosa in the Danish Neolithic age". Danish Medical Bulletin 19(5): 176-180.

Sallustio, C.; Zaio, P. y Rubini, M. 2004: “Un probabile caso di tubercolosi ossea in una sepoltura dell'Eta del Bronzo Medio". Archivio per l'Antropologia e la Etnologia 134: 223-135.

Santos, A. L. y Roberts, C. A. 2006: "Anatomy of a serial killer: Differential diagnosis of tuberculosis base on rib lesions of adult individuals from the Coimbra
Identified Skeletal Collection, Portugal". American Journal of physical Anthropology 130 (1): 38-49.

Santos, A. L. y Suby, J. A. 2012: "Tuberculosis en retrospectiva: revisión de los conocimientos actuales y su aplicación en el estudio de restos humanos". Cuadernos de Prehistoria y Arqueología de la Universidad de Granada 22: 127-148.

Scheuer, L. y Black, S. 2000: Developmental juvenile osteology. Academia Press. San Diego.

Spekker, O.; Pálfi, G.; Kozocsay, G.; Pósa, A.; Bereczki, Z. y Molnár, E. 2012: "New cases of probable skeletal tuberculosis from the Neolithic period in Hungary. A morphological study". Acta Biologica Szegediensis 56 (2): 115-123.

Steinbock, R. T. 1976: Paleopathological diagnosis and interpretation. Bone diseases in ancient human populations. Charles C. Thomas. Illinois.

Todd, T. W. 1920: "Age changes in the pubic bone: The white male pubis". American Journal of Physical Anthropology 3: 427-470.

Ubelaker, D. H. 1984: Human skeletal remains: excavation, analysis, interpretation. Taraxacum. Washington, DC.

Wirth, T.; Hildebrand, F.; Allix-Béguec, C.; Wölbeling, F.; Kubica, T.; Kremer, K. y Niemann, S. 2008: “Origin, spread and demography of the Mycobacterium tuberculosis complex". PLoS Pathogens 4(9): 1-10.

Zamora, A. 1976: Excavaciones de la Cueva de La Vaquera, Torreiglesias, Segovia (Edad del Bronce). Publicaciones históricas de la Excma. Diputación Provincial de Segovia. Segovia. 